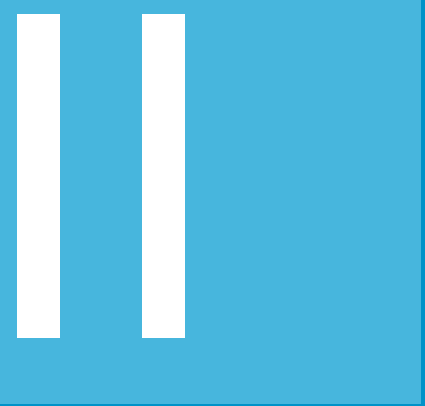

\title{
Los valores personales priorizados por los profesionales adventistas en el Perú
}

\section{Personal values prioritized by the Adventist professionals in Peru} Universidad de Montemorelos, México

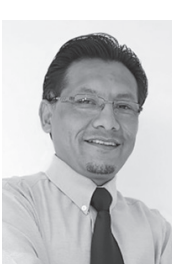

\section{Juan Carlos Niño de Guzmán Miranda}

Licenciado en Administración. Magister en Marketing y Negocios Internacionales y Doctor en Administración. Realizó actividades de docencia y gestión en su alma mater, la Universidad Peruana Unión. Asimismo, es ponente en eventos de índole nacional e internacional. Actualmente se desempeña como docente de pregrado y posgrado de la Facultad de Ciencias Empresariales y Jurídicas de la Universidad de Montemorelos (México).

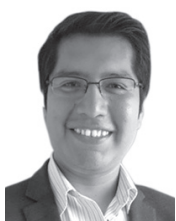

\section{Edison Effer Apaza Tarqui}

Ingeniero en Estadística e Informática y Especialista en Estadística Aplicada para Investigación. Docente de la Universidad Peruana Unión, actualmente laborando como Coordinador del Curso de Investigación en Ciencias Empresariales, en la Facultad de Ciencias Empresariales de la Universidad Peruana Unión. 


\section{Resumen}

El objetivo de esta investigación consiste en describir los valores personales que son priorizados por los profesionales adventistas en el Perú. La investigación tiene un diseño no experimental y es transversal. Para la recolección de información se aplicó la Encuesta de Valores Personales en una muestra de 250 profesionales adventistas que se distribuyen en el territorio peruano. Asimismo, se presentó el cuestionario a profesionales adventistas ubicados cerca de los tres campus (Lima, Juliaca y Tarapoto) de la Universidad Peruana Unión. La encuesta mide cuatro dimensiones de los valores personales: Búsqueda de Logro, Búsqueda de Placer, Honestidad y Respeto. Los resultados demuestran que los valores personales más priorizados por los profesionales adventistas se encuentran en la dimensión de Honestidad; para demostrar esto se realizó un análisis factorial confirmatorio, el mismo que permitió identificar a dos grupos marcados en la muestra, los de "Identidad posmodernista" y los de "Identidad adventista", estos últimos tienen un comportamiento más conservador y tienen muy internalizados los valores de la sinceridad, confianza, integridad, armonía, reciprocidad, responsabilidad, honor, autoestima, tolerancia, aprecio, perseverancia, entre otros. En conclusión, los valores personales practicados con mayor realce, por los profesionales adventistas son la Honestidad y la Búsqueda de Logro; sin embargo, vale resaltar que el Respeto es uno de los valores que se necesita mejorar, y que existe un segmento de profesionales adventistas que tienen una tendencia a priorizar el Placer.

Palabras clave: valores personales, profesionales adventistas, perfil empresario adventista

\section{Abstract}

The purpose of this research is to describe the personal values that are prioritized by the Adventist professionals in Peru. Research has an experimental design and is not cross. For data collection Personal Values Survey in a sample of 250 Adventist professionals distributed in Peruvian territory it was applied. In addition, the questionnaire was presented to Adventist professionals located near the three campuses (Lima, Juliaca and Tarapoto) of the Peruvian Union University. The survey measures four dimensions of personal values: Achievement search, search Placer, honesty and respect. The results show that the personal values more prioritized by the Adventist professionals are in the dimension of Honesty; to demonstrate that a confirmatory factor analysis was performed, the same as identified two marked groups in the sample, of "postmodernist Identity" and those of "Adventist Identity", the latter have a behavior more conservative and have very internalized values sincerity, trust, integrity, harmony, reciprocity, responsibility, honor, esteem, tolerance, appreciation, perseverance, among others. In conclusion, personal values practiced with greater prominence, by Adventist professionals are honesty and the Pursuit of Achievement; however, worth noting that Respect is one of the values that need to be improved, and that there is a segment of Adventist professionals who have a tendency to prioritize pleasure.

Keywords: Personal values, adventist professionals 


\section{Introducción}

En la actualidad, la sociedad viene siendo protagonista de cambios en diversos aspectos, en lo social, en lo político, en lo económico, en lo medioambiental, entre otros. Algunos cambios buscan el bienestar común, pero otros no ayudan a mejorar la situación de los ciudadanos. Para que hayan cambios, se toman decisiones y estas se hacen en base a los valores personales que tienen las personas.

Acerca de los valores personales, Toffler (1993, p.17), hace una declaración muy acertada en su obra La Tercera Ola:

Una poderosa marea se está alzando hoy sobre gran parte del mundo, creando un nuevo, y a menudo extraño entorno en el que trabajar, jugar, casarse, criar hijos o retirarse ha quedado atrás. En ese desconcertante contexto, los hombres de negocios nadan contra corrientes económicas sumamente erráticas; los políticos ven violentamente zarandeadas sus posiciones; universidades, hospitales y otras instituciones luchan desesperadamente contra la inflación. Los sistemas de valores se resquebrajan y hunden, mientras los salvavidas de la familia, la Iglesia y el Estado, cabecean a impulsos de tremendas sacudidas".

Sin duda, esta descripción que hacía Toffler era con referencia a lo que hoy se denomina "globalización".

Mientras la globalización ha ido insertándose en diversas latitudes, en lo social, sin el ánimo de impulsar el machismo y tal como Giddens lo describe, la familia ya no es la misma, una de sus razones se basa en que la mujer se insertó en el proceso productivo (Infante, 2007, pp. 55-65) .

En lo político, Garza (2005, pp.155-171), sostiene que un rasgo distintivo de la globalización, es la pérdida de los valores humanos, así como el debilitamiento del Estado-nación, que es la base de las instituciones jurídico políticas de lo que se conoce como Estado de derecho. En la misma obra de este autor se menciona que Baudrillard también hace énfasis en que se ha quebrado el espejo de la universalidad: "La situación cambia y se radicaliza con la pérdida de autoridad y de legitimidad de los valores". Ejemplos de esto, se pueden ver en las noticias actuales provenientes de Brasil, de Siria, de Venezuela, de España, entre muchos otros.

En lo económico, la situación en la que se encuentran algunos países es demasiado delicada. Al respecto, Martínez (2014, pp.5-6), identifica tres tipos 
Juan Carlos Niño de Guzmán Miranda -

Edison Effer Apaza Tarqui

de pensamiento, el primero que valora muy positivamente el capitalismo como un sistema capaz de acercar los productos a casi todo el mundo y esto significa un buen avance para calidad de vida de la población en general; el segundo que el capitalismo no es sostenible, que mejor es una economía basada en el bien común que tampoco ha tenido muy buenos ejemplos al momento de demostrar su practicidad; y el tercer grupo que valora el capitalismo negativamente calificándolo de "casino global"en el que los Estados y la política pierden protagosnismo a favor de los grandes poderes económico financieros, a la vez, este grupo promueve la "economía social de mercado"; sin embargo, a pesar de haberse intentado converir una de estas tres formas de pensar en la ideal, no se ha podido hasta hoy en vista que casos escandalosos como la crisis financiera del 2007 denominada "burbuja financiera" en el que bancos, aseguradoras, inmobiliarias y otras compañías provocaron un déficit financiero que contrajeron quiebras de empresas y su consecuente generación de desempleo en lugares impensados; o casos como el de la Federación Internacional de Futbol Asociado (FIFA), Panama Papers, u otros relacionados con mafias y "mercados negros" que se desarrollan al margen de la ley.

En el caso del medio ambiente, existe también una crisis generalizada, los acuerdos principalmente promovidos por la ONU, entre los más recientes se encuentran el protocolo de Kyoto (1997) la Conferencia de las Partes COP desde Berlín en 1995 hasta París en 2015 (Romero, 2014), son ambiciosos planes con los que se promueve la lucha contra el cambio climático. Sin embargo, los derrames de petróleo, el abuso de la explotación minera con el respectivo daño por los relaves y la aparición de mineros informales; no ayudan en el avance de los objetivos trazados, los animales que viven en estado salvaje y que son víctimas de la caza furtiva para explotarlos mediante la venta clandestina, la tala indiscriminada de bosques en países donde reina la impunidad, entre otros casos que pueden describir a personas que han dejado de lado la práctica de valores personales.

En vista de esta realidad y siendo que se asume que aquellas personas que han sido formadas profesionalmente en centros de enseñanza de nivel superior, de quienes se espera un rol protagónico para liderar cambios en pro de una mejor condición medioambiental se presenta la oportunidad de realizar una investigación entre los profesionales cristianos, específicamente aquellos que profesan la fe adventista en el territorio peruano. De esta manera, el objetivo trazado para este trabajo consiste en identificar los valores personales que son priorizados por los profesionales adventistas en el Perú. 


\section{Materiales y métodos}

El diseño utilizado es no experimental y transversal. El medio en el que se realizó es el territorio peruano en el primer semestre del año 2015. Para esta investigación se realizó un muestreo probabilístico aleatorio simple equivalente a 250 personas, este se tomó de una población de 1481 profesionales adventistas que practican valores personales. Se hace este estudio en una muestra de los profesionales adventistas del Perú puesto que se trata de personas preparadas, con capacidad de decisión que además practican cotidianamente los valores personales basados en el cristianismo y cuyo proceso de toma de decisiones resulta de interés para el investigador. Asimismo, se consideró recoger información en las ciudades de Lima, Tarapoto y Juliaca, lugares donde radica una mayor población de profesionales adventistas debido a la ubicación de los campus universitarios de la Universidad Peruana Unión, alma mater de la mayoría de ellos.

Algunos profesionales adventistas, conformantes de la muestra, han tenido dificultades para poner en práctica sus valores personales en su centro de labor debido a costumbres y prácticas contrarias a las de ellos como pueden ser la corrupción de funcionarios, el tráfico de influencias, la malversación de fondos, la evasión de impuestos, entre otros.

Por el contrario, otros profesionales adventistas han encontrado apoyo en la comunidad y en su centro de labor frente a actividades promovidas por ellos, como son: la donación de sangre, la promoción y práctica de un estilo de vida saludable, las colectas públicas para el auxilio ante desastres, actividades para promover el cuidado del medio ambiente y la educación basada en valores cristianos (entre estos el respeto, la honestidad, la solidaridad, la libertad, la esperanza, etc.). La técnica para este trabajo fue la encuesta.

El instrumento "Encuesta de valores personales" consta de 32 valores que normalmente son practicados por los profesionales adventistas y que fueron tomados del instrumento Rokeach Values Survey (Rokeach, 1973) y adaptados a esta encuesta, en una escala de medición de importancia de 1 al 10, siendo uno el menos importante y el 10 el más importante. Estos valores forman parte de 4 dimensiones que son:

\section{a) Búsqueda de Logro:}

Valor natural perseguido por las personas con el fin de alcanzar lo que se intenta o desea. Este valor contiene otros sub-valores, los cuales son: perseverancia, deseo, capacidad, mente abierta, sabiduría, reconocimiento social e intelecto. 
Juan Carlos Niño de Guzmán Miranda -

Edison Effer Apaza Tarqui

\section{b) Búsqueda de Placer}

Valor perseguido por las personas para sentir satisfacción, una sensación agradable producida por la realización o suscepción de algo que gusta o complace. Asimismo, se entiende como el sentimiento de complacencia en la posesión, recuerdo o esperanza de bienes o cosas apetecibles. Este valor tiene otros subvalores que son: felicidad, seguridad familiar, una vida confortable, igualdad, placer, un mundo de belleza, libertad y armonía interior.

\section{c) Honestidad}

Es el pilar en el que reposa la rectitud. Ser honesto es una virtud, un modo de comportarse, valor que asume la persona para lograr la realización de su vida, su ideal. La honestidad es como la honradez, requieren de coherencia total entre lo que se dice y lo que se hace. Va de la mano con la verdad, ser honesto es ser verídico, no tienen posibilidad de división. Contiene los sub-valores: integridad, verdad, confianza, sinceridad y honor.

\section{d) Respeto}

Valor que comienza en la propia persona, se basa en reconocer el propio ser como entidad única. Es imprescindible para lograr las relaciones de convivencia en cualquier ámbito donde se desenvuelve el individuo. Es el reconocimiento y la valoración del otro como igual con las características que le son propias. Es fruto del diálogo ya que, aunque las ideas sean diferentes, cuando se establece es posible compaginar las posturas, que las sostienen. Una relación respetuosa permite establecer el diálogo. Es un valor que facilita al ser humano reconocer, aceptar, apreciar y valorar las cualidades de los demás, ya sea por su conocimiento, experiencia, valor como persona o capacidades diferentes. Este concepto conlleva los siguientes sub-valores: aceptación, tolerancia, responsabilidad, reciprocidad y aprecio.

\section{Confiabilidad y Validez del Instrumento}

Se efectuó el análisis de confiabilidad a partir de los datos obtenidos con la muestra piloto, para ello se utilizó el estadístico Alfa de Cronbach y se obtuvieron los resultados siguientes: 
Tabla 1

Confiabilidad del instrumento Valores personales y sus respectivas dimensiones

\begin{tabular}{lccc}
\hline & $\begin{array}{c}\text { Número de } \\
\text { Ítems }\end{array}$ & $\begin{array}{c}\text { Alfa de } \\
\text { Cronbach }\end{array}$ & N \\
\hline Búsqueda de logro & 9 & 0.872 & 250 \\
Búsqueda de placer & 10 & 0.832 & 250 \\
Honestidad & 5 & 0.671 & 250 \\
Respeto & 8 & 0.892 & 250 \\
Valores personales & 32 & 0.945 & 250 \\
\hline
\end{tabular}

El coeficiente Alfa de Cronbach para el instrumento Valores Personales es de .945 , cercano a la unidad; por lo que denota una elevada confiabilidad. Así también las dimensiones superan el límite establecido, para considerarlas confiables.

Asimismo, se realizó el análisis factorial utilizando el Coeficiente de KMO y la prueba de Bartlett de la escala Valores Personales, los resultados obtenidos son:

Tabla 2

Prueba KMO para la validez del instrumento de Valores personales

\begin{tabular}{|c|c|c|}
\hline \multicolumn{3}{|c|}{ KMO y prueba de Bartlett } \\
\hline \multicolumn{2}{|c|}{ Medida de adecuación muestral de Kaiser-Meyer-Olkin. } & 940 \\
\hline \multirow[t]{3}{*}{ Prueba de esfericidad de Bartlett } & Chi-cuadrado aproximado & 5957,514 \\
\hline & GI & 496 \\
\hline & $\mathrm{p}$ & ,000 \\
\hline
\end{tabular}

El instrumento presenta validez debido a que el índice KMO es de .940. Al final del procesamiento de los datos se realizó un análisis factorial confirmatorio para segmentar los principales valores personales que tienen internalizados los profesionales adventistas. 
Juan Carlos Niño de Guzmán Miranda -

Edison Effer Apaza Tarqui

\section{Resultados y discusión}

Según las dimensiones de Valores Personales, se encontró:

Tabla 3

Niveles de los valores personales y sus respectivas dimensiones

\begin{tabular}{lcccccccc}
\hline & \multicolumn{2}{c}{ Bajo } & \multicolumn{2}{c}{ Medio } & \multicolumn{2}{c}{ Alto } & \multicolumn{2}{c}{ Total } \\
\hline Valores Personales & 76 & $30,4 \%$ & 99 & $39,6 \%$ & 75 & $30,0 \%$ & 250 & $100,0 \%$ \\
Búsqueda de Logro & 78 & $31,2 \%$ & 103 & $41,2 \%$ & 69 & $27,6 \%$ & 250 & $100,0 \%$ \\
Búsqueda de Placer & 79 & $31,6 \%$ & 104 & $41,6 \%$ & 67 & $26,8 \%$ & 250 & $100,0 \%$ \\
Honestidad & 77 & $30,8 \%$ & 73 & $29,2 \%$ & 100 & $40,0 \%$ & 250 & $100,0 \%$ \\
Respeto & 87 & $34,8 \%$ & 98 & $39,2 \%$ & 65 & $26,0 \%$ & 250 & $100,0 \%$ \\
\hline
\end{tabular}

\section{Búsqueda de Logro}

El $41.2 \%$ de los encuestados indica que tiene un nivel medio, lo que significa que no está desarrollado del todo este valor ni es deficiente.

Se puede deducir que o no alcanzan su máximo nivel de performance por falta de oportunidades o son conformistas con lo que han logrado hasta ahora. Para el caso de los profesionales con mayor edad, puede que se deba a que ya no tienen mayores anhelos de logro (estatus laboral, estatus académico e ingresos económicos).

\section{Búsqueda de Placer}

Llama la atención que el $41.6 \%$ de la muestra refiere que tiene un nivel de búsqueda de placer y tiende a la baja, siendo el segundo lugar con un $31.6 \%$ y de lo cual se deduce que el profesional adventista no busca el placer en su mayoría, es decir, el $73.2 \%$ no prioriza la búsqueda de placer. Sin embargo, hay un $26,8 \%$ que considera como importante este valor, por lo que se asume que la generación de profesionales adventistas jóvenes tiende a buscar valores como la libertad, felicidad, armonía interior, un mundo de belleza, una vida confortable, entre otros.

\section{Honestidad}

Este valor tiene un $40 \%$ de prioridad alta y una prioridad media de 29. $2 \%$ entre los profesionales adventistas, de lo que se puede inferir que ser transparente o íntegro es primordial en su vida profesional y cotidiana. 


\section{Respeto}

El 39,2\% de esta dimensión lo ubica como 'medio', lo que significa que el nivel de importancia en cuanto al respeto es moderado con tendencia a minimizar los valores de tolerancia, aceptación, responsabilidad y reciprocidad la cual puede ser mejorada.

Se puede deducir que el posmodernismo (hedonismo, consumismo, etc.) amenaza con ingresar en el estilo de vida del profesional adventista.

\section{Identidad posmodernista vs Identidad adventista}

Por otro lado, en el análisis factorial confirmatorio que se realizó sobre la escala de Valores Personales, se encontró que existen dos grupos bien marcados a los cuales se les denominó en esta investigación como: "Identidad posmodernista" e "Identidad adventista".

Por un lado está la "Identidad posmodernista" caracterizada por la cultura de valores mucho más liberales y basada en el posmodernismo que se caracteriza por promover entre las personas algunos principios emergentes que incluyen el rechazo a la metanarrativa, la afirmación del pluralismo, el contextualismo y el constructivismo, y la celebración de la diversidad. Asimismo, resalta el individualismo, el humanismo secular, haciendo del hombre como la medida y el punto de referencia para todas las cosas; el racionalismo y el economicismo que impulsa la popularidad de algunos modelos socioeconómicos, incluyendo el capitalismo, y ha favorecido el desarrollo de tendencias hedonísticas y narcisistas, muy divulgadas en la sociedad (Taylor, 2012, pp. 85-100). Esta identidad impulsa el egoísmo.

En este grupo denominado "Identidad posmodernista", como se puede (Figura 1), se encuentran los valores de placer, belleza, una vida emocionante, una vida confortable, reconocimiento, ser intelectual, ser imaginativo, sentirse independiente, un mundo en paz, y una mente abierta; y haciendo una análisis de las características sociodemográficas de quienes dan prioridad a estos valores, se encuentran más varones y profesionales jóvenes en esta lista. Esto significa que la tendencia a prácticas posmodernistas está latente, y tiene su explicación en el estilo de vida que se lleva en las grandes ciudades, el cual promueve el consumismo y hedonismo, convirtiendo con esto a muchos profesionales en mano de obra que solamente genera ingresos para tener la posibilidad de consumir aquellos bienes o servicios que deben ser adquiridos o usados según lo dicta la sociedad actualmente.

Por otro lado, está la "Identidad adventista" cuyas características se identifican con una cultura conservadora que prioriza otros valores como: la 
Juan Carlos Niño de Guzmán Miranda -

Edison Effer Apaza Tarqui

sinceridad, integridad, confianza, verdad, armonía, reciprocidad, responsabilidad, honor, autoestima, sabiduría, seguridad, aceptación, tolerancia, sentirse apreciado, perseverancia, felicidad, logro, sentirse capaz, ser emprendedor, igualdad, libertad y alegría.

La filosofía de la "Identidad adventista", que tiene una cosmovisión cristiana, da por hecho en que la verdad está en las Sagradas Escrituras, la Biblia, que es la palabra de Dios, ésta promueve los valores morales y contribuye a formar un sentido personal de identidad, dirección y pertinencia; extendiendo con ello un llamado para la justicia, la sensibilidad y la compasión. Asimismo, la cosmovisión cristiana es capaz de promover un marco ético a la persona, lo cual brinda estabilidad y seguridad (Taylor, 2012, pp. 85-100).

Se puede destacar que en este grupo de la "Identidad adventista" con claridad que (Figura 1) existe una gran diferencia con la "Identidad posmodernista", con respecto al tipo de valores que practican. Mientras en uno, se promueve la verdad, la integridad, la tolerancia y vida en comunidad, en el otro se prioriza la individualidad y el hedonismo teniendo al placer como valor priorizado.

Significa que los profesionales adventistas, de alguna manera contribuyen con su práctica de valores personales a generar una corriente conciliadora que comparte un estilo de vida con mentalidad positiva y buscando ayudar a las personas a tener un sentido de vida, motivos para ayudar a otros y vivir en comunidad.

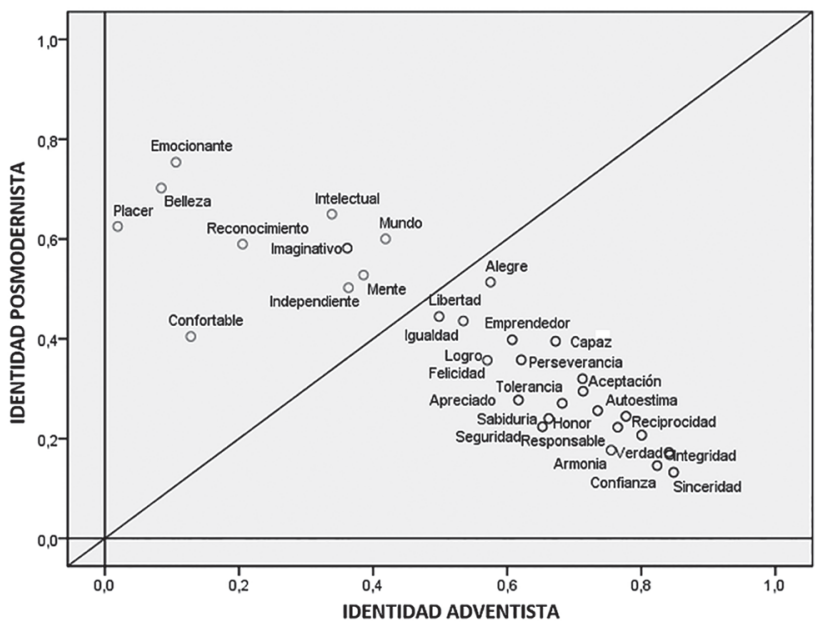

Figura 1. Segmentación de Valores Personales 


\section{Conclusiones y recomendaciones}

1. Los valores personales priorizados por los profesionales adventistas están organizados en cuatro dimensiones, los mismos que tienen el sigiuente orden: Honestidad $40 \%$, en el nivel alto, seguido de la Búsqueda de Logro con un $27,6 \%$ en el nivel alto, así también la Búsqueda de Placer con un $26,8 \%$ en el nivel alto y finalmente el Respeto con un $26 \%$ en el nivel alto. De esta manera se concluye que los valores más importantes por los profesionales adventistas son la Honestidad, y la Búsqueda de Logro.

2. En un nivel medio o que regularmente toma en consideración el profesional adventista con respecto a los valores personales están: La Búsqueda de Placer en un 41,6 \%, así también la Búsqueda de Logro con 41,2 \%, seguido del Respeto con un $39,2 \%$ y finalmente la Honestidad con $29,2 \%$. Se concluye que los valores que resaltan en un nivel medio considerados por los profesionales adventistas son la Búsqueda de Placer y la Búsqueda de Logro.

3. De acuerdo a los valores personales, las dimensiones con un nivel bajo son el Respeto con un 34,8\%, seguido de la Búsqueda de Placer con un 31,6 $\%$, así también la Búsqueda de Logro con un 31,2 \% y finalmente la Honestidad con 30,8 \%. Se concluye que la Búsqueda de Placer es un valor de no mucha importancia y hay una amenaza para los profesionales adventistas por la poca priorización del Respeto, cosa que se sugiere revertir.

4. Independientemente, se encontró que los valores con mayor realce en la práctica de los profesionales adventistas son la Honestidad y la Búsqueda de Logro. El valor que necesita mejorar es el Respeto y vale mencionar que existe un segmento de profesionales adventistas que Buscan el Placer.

5. Otra contribución presente en la investigación es la confiabilidad del instrumento adaptado, así como la validez del mismo, la cual demostró ser meritoria para reafirmar el uso del Instrumento denominado 'Valores personales' y ser aplicado en poblaciones similares.

Juan Carlos Niño de Guzmán Universidad de Montemorelos, México email: jcnino@um.edu.mx

Recibido: 11 de enero de 2016 Aceptado: 15 de marzo de 2016 
Juan Carlos Niño de Guzmán Miranda -

Edison Effer Apaza Tarqui

\section{Referencias}

Garza, J. (2005) Crisis del estado y los valores humanos. BJVIIJ-UNAM. http://biblio.juridicas.unam. $\mathrm{mx} /$ libros/6/2990/10.pdf

Infante, J. (2007) Anthony Giddens, una interpretación de la globalización. Revista Trayectorias. UANL, México, 2007 (p. 55 - 65)

Martínez,Emilio. (2014) Ética y crisis (económica). VII Informe sobre exclusión y desarrollo social en España 2014. Universidad de Murcia. España.

Rokeach, M. (1973). The nature of of Human Values. New York: Free Press.

Romero, Freddie A. (2014) Historia de conferencias climáticas, COP y CMP. https:// opinionyaccion. wordpress.com/2014/11/23/historia-de-conferencias-climaticas-cop-y-cmp/

Toffler, Alvin. (1993) La tercera ola. Edivisión, México, (p.17)

Taylor, J. W. (2012). Posmodernidad y educación cristiana: Desafíos ideológicos contemporáneos. (Enfoques, pp.85-100). 